OPEN

SUBJECT AREAS:

VISUAL SYSTEM

COGNITIVE AGEING

Received

9 June 2014

Accepted

17 November 2014

Published

4 December 2014

Correspondence and requests for materials should be addressed to

M.K. (m.kunchulia@ agruni.edu.ge)

\title{
Small effects of smoking on visual spatiotemporal processing
}

\author{
Marina Kunchulia' ${ }^{1}$ Karin S. Pilz ${ }^{2} \&$ Michael H. Herzog ${ }^{3}$
}

${ }^{1}$ Institute of Cognitive Neurosciences, Agricultural University of Georgia, Tbilisi, Georgia, ${ }^{2}$ School of Psychology, University of Aberdeen, Scotland, UK, ${ }^{3}$ Laboratory of Psychophysics, Brain Mind Institute, School of Life Sciences, Ecole Polytechnique Federale de Lausanne (EPFL), Switzerland.

Nicotine is an important stimulant that is involved in modulating many neuronal processes, including those related to vision. Nicotine is also thought to play a key role in schizophrenia: A genetic variation of the cholinergic nicotine receptor gene, alpha-7 subunit (CHRNA7) has been shown to be associated with stronger backward masking deficits in schizophrenic patients. In this study, we tested visual backward masking in healthy smokers and non-smokers to further understand the effects of nicotine on spatiotemporal vision. In the first study, we tested 48 participants, a group of non-smokers $(n=12)$ and three groups of regular smokers that were either nicotine deprived $(n=12)$, non-deprived $(n=12)$ or deprived but were allowed to smoke a cigarette directly before the start of the experiment $(n=12)$. Performance was similar across groups, except for some small negative effects in nicotine-deprived participants. In the second study, we compared backward masking performance between regular smokers and non-smokers for older $(n=37,13$ smokers) and younger ( $n=67,21$ smokers) adults. Older adults performed generally worse than younger adults but there were no significant differences in performance between smokers and non-smokers. Taken together, these findings indicate that nicotine has no long-term negative effects on visual spatiotemporal processing as determined by visual backward masking.

$\mathrm{N}$ icotine is a psychoactive component of tobacco that affects the central nervous system and is the third most prevalent freely accessible drug after alcohol and caffeine. Nicotine has many negative effects on health as, for example, an increased risk for stroke, cancer, heart diseases and a variety of ocular diseases $^{1-7}$. Interestingly, however, nicotine has been shown to have positive effects on cognition and it has been suggested that nicotine addiction may partly relate to its cognitive-enhancing effects ${ }^{8-12}$. For example, nicotine has been shown to improve memory functions ${ }^{13-15}$ and smokers have reported beneficial effects of smoking on concentration and memory ${ }^{16-18}$. In addition, it has been shown that nicotine abstinence in smokers is associated with decreased cognitive functions including impairments of sustained attention and poorer working memory ${ }^{19-24}$.

The effects of smoking on vision are largely understudied and to the best of our knowledge, no study has tested whether and how nicotine affects visual temporal processing or the relationship between nicotine addiction and visual temporal processing.

A powerful paradigm that is often used to study visual temporal processing is visual backward masking: A target stimulus is followed by a mask that alters the visibility of the target. A very sensitive variation of backward masking is the shine through masking paradigm ${ }^{25-27}$. In this paradigm, participants have to discriminate the offset direction of a vernier stimulus that precedes a mask grating of 25-elements (Figure 1). A vernier stimulus consists of two abutting bars, of which the lower bar can be offset to the right or left. When masked with a 25-element grating, the vernier is perceived as being wider and brighter than it really is and appears to be superimposed on the grating (Figure 1). The vernier duration and the spatial offset between the two bars are adjusted individually before adding a mask so that participants can easily discriminate the offset direction. The stimulus onset asynchrony (SOA) between vernier and grating is then used as a measure for the strength of the masking effect: Longer SOAs indicate strong masking effects, whereas shorter SOAs indicate weaker masking effects. If the grating has only 5 -elements, shine-through does not occur and the vernier is almost invisible even though the 5 -element grating is contained within the 25-element grating. Only when the SOA is increased for the 5element grating, the vernier becomes visible again, indicating stronger masking effects for the 5-element than the 25-element grating. Shine-through is affected by both temporal and spatial alterations of the grating. For example, when adding spatial inhomogeneities, such as gaps close to the center of the 25 -element grating, the visibility of the vernier is strongly diminished ${ }^{25,26}$. Likewise, temporal alterations such as inserting a 5-element 
A.

$$
\text { I }
$$

ISI
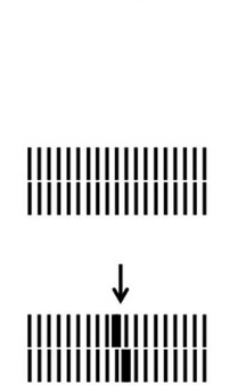

B.
C.<smiles>II</smiles>

ISI
I

ISI
1

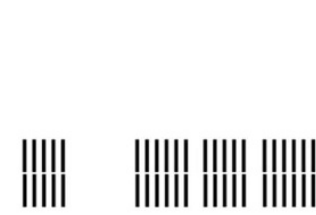

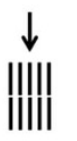

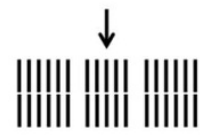

D.

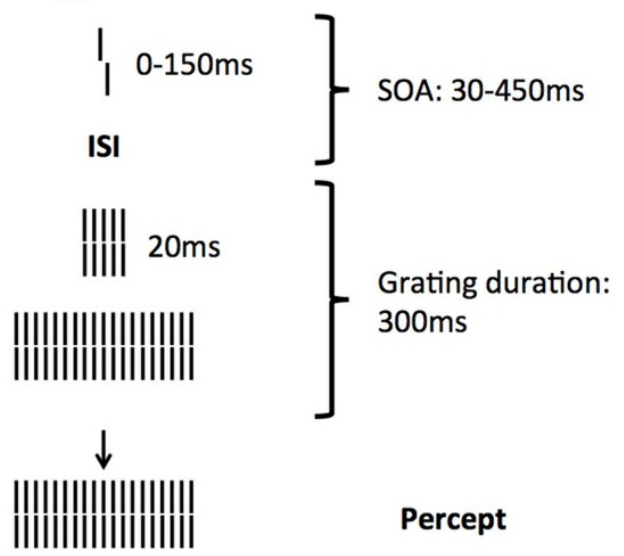

Figure $1 \mid$ Shine-through. A vernier is presented for a short time and followed by a grating comprising more than 25 elements. The foregoing vernier appears to be superimposed on the grating, looking wider, brighter, and for some observers, even longer. (B) For a grating with five elements, the visibility of the preceding vernier is strongly diminished. (C) Visibility is also strongly diminished if the extended grating contains gaps (gap grating) or (D) a fiveelement grating presented for 20 ms precedes the 5-25-elements grating (see scale on the right for timing in this condition). The interstimulus interval (ISI) denotes the time difference between vernier offset and grating onset, while the stimulus onset asynchrony (SOA) denotes the difference between vernier and grating onsets. The bottom row shows the percept. The vernier offset shown is strongly exaggerated. The spacing between grating elements is $200^{\prime \prime}$, whereas the vernier offsets are often much smaller. Figure adapted from Herzog et al. ${ }^{30}$

grating between the vernier and the larger grating can dramatically deteriorate performance ${ }^{27}$. The shine-through effect is related to the grouping of the mask: Only if the grating is perceived as a homogeneous and extended object, shine-through occurs. However, if spatial or temporal inhomogeneities segment the extended grating into smaller parts, the shine-through effect is decreased or even extinguished (Figure 1).

The shine-through paradigm is a powerful tool to assess visual spatiotemporal processing in special populations. For example, Overney et al. ${ }^{28}$ showed enhanced temporal processing in tennis players compared to non-athletes and triathletes and Kunchulia et al. ${ }^{29}$ found that alcohol strongly deteriorates performance. In addition, there are tremendous changes in schizophrenic patients who show increases in SOA of about a factor of five compared to healthy controls and interestingly, also the healthy relatives of the patients are affected ${ }^{30,31}$.

Bakanidze et $\mathrm{al}^{32}$ found a direct association between a genetic variation of the cholinergic nicotine receptor gene, alpha-7-subunit (CHRNA7) and the shine-through effect in schizophrenic patients. These results are in accordance with the hypothesis that the nicotinic system plays a key role in the pathophysiology of schizophrenia ${ }^{33-35}$. Many schizophrenic patients are heavy smokers ${ }^{36-39}$ and smoking has been related to attentional enhancements in schizophrenic patients $^{39}$ supporting the idea that smoking is a form of selfmedication $^{39-43}$.

The purpose of this study was two-fold: First, we were interested in the effects of smoking on spatiotemporal processing in general and, second, we were particularly interested whether nicotine has similar effects on backward masking as it has been found in schizophrenic patients. In short, the reasoning is as follows: Shine-through is diminished in schizophrenia. The nicotine system has been directly linked to schizophrenia and many schizophrenic patients are heavy smokers, which is thought to be a form of self-medication. Nicotine has been shown to have cognitive-enhancing effects. Here we tested whether nicotine also has positive affects on visual processing in healthy participants. In part one, we compared performance of non-smokers to smokers, deprived smokers and smokers who were allowed to smoke a cigarette directly before the experiment. Since smoking seems to have positive effects in schizophrenia, we expected improvements in backward masking for regular smokers and deprived smokers that were allowed to smoke a cigarette directly before the experiment compared to non-smokers and deprived smokers $^{30,31}$.

It has been shown that smoking increases the risk for developing Alzheimer's disease and age-related cognitive decline ${ }^{44}$. In addition, smoking has been shown to affect age-related eye diseases including age-related macular degeneration ${ }^{45}$. Interestingly, nicotinic acetylcholine receptors have been found to decline with $\operatorname{age}^{46}$. Consequently, the effects of smoking on backward masking might change during ageing. Therefore, in order to investigate the longterm influence of smoking on backward masking, we compared healthy younger and older smokers and non-smokers in part two of the study. In both parts of the study, effects were relatively small.

\section{Results}

There were no significant differences for vernier duration (VD), stimulus onset asynchrony for the 5-element grating (SOA5), or the 25-element grating (SOA25) between men and women and therefore, results for both sex groups are reported together.

Vernier duration. In part one of the study, vernier duration (VD), the duration of the vernier at which participants could discriminate the offset at $75 \%$ correct, was similar for all participants. All subjects had a vernier duration of $20 \mathrm{~ms}$, apart from one male and one female subject in the deprived + group who had a vernier duration of $30 \mathrm{~ms}$.

In part two of the study, a 2(Age) x 2 (Smoking) ANOVA showed a main effect of Age (older adults: $\mathrm{M}=113.2, \mathrm{SD}=147.2$; younger adults: $\mathrm{M}=30.7, \mathrm{SD}=42.6 ; \mathrm{F}(1,100)=18.2, \mathrm{p}<0.001)$. The vernier durations for younger adults in part two of the study were notably larger than in part one. This was mainly due to four younger participants having vernier durations of $150 \mathrm{~ms}$ or above and one with a vernier duration of $50 \mathrm{~ms}$. All other younger participants $(n=62)$ had a vernier duration of $20 \mathrm{~ms}$. There were no other main effects or interactions (Figure 2, left). Overall, only five out of 67 young participants had a VD larger than $20 \mathrm{~ms}$, whereas 20 out of 37 older participants had a VD larger than $20 \mathrm{~ms}$.

Backward Masking. In part one of the study, ANOVAs on the factor Group showed no significant difference between groups for SOA25 $(\mathrm{F}(3,44)=0.6, \mathrm{p}=0.6)$ and only a marginal difference for SOA5 $(\mathrm{F}(3,44)=2.7, \mathrm{p}=0.055)$ as shown in Figure 3 (left). Post-hoc tests showed that deprived smokers had stronger masking effects than 

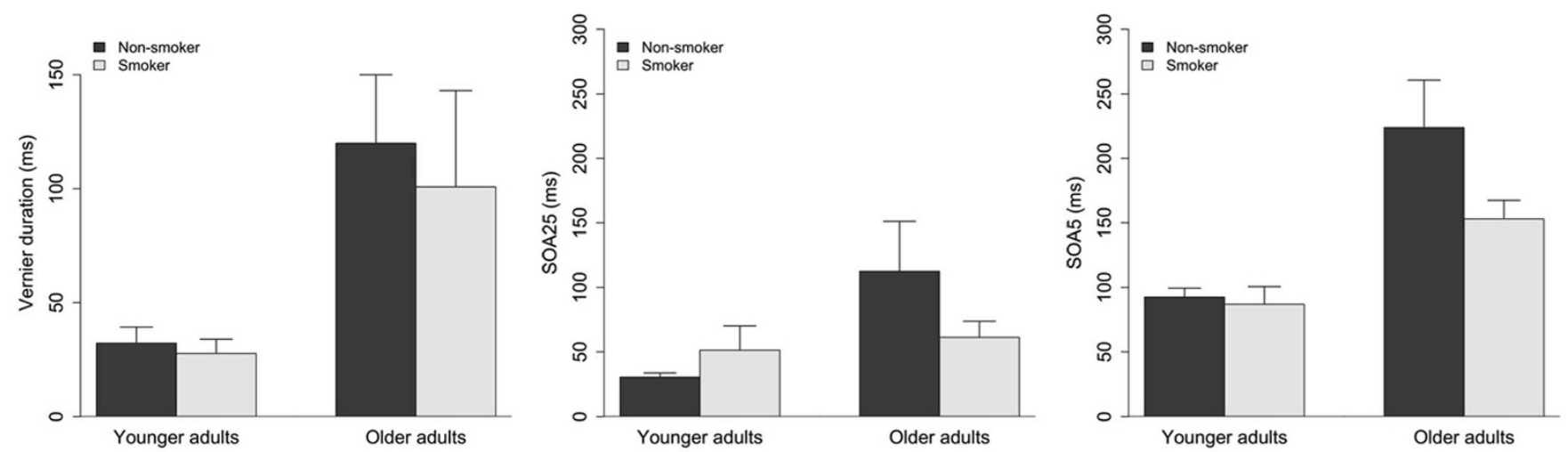

Figure 2 Vernier duration for older and younger smokers and non-smokers in part two of the study (left). Error bars depict standard error of the mean (SE). There was only a significant difference between age-groups but no main effect of Smoking and no Age x Smoking interaction. SOAs for 5 (middle) and 25- element gratings (right) for older and younger smokers and non-smokers in part two of the study. Error bars depict standard errors of the mean (SE). There was only a significant difference between age-groups but no main effect of Smoking and no Age $\mathrm{x}$ Smoking interaction interaction.

deprived + smokers $(\mathrm{t}(22)=2.8, \mathrm{p}=0.006$; Bonferroni corrected alpha of $0.008(0.05 / 6))$. There was no other significant difference with $\mathrm{p}>0.3$ for all other comparisons.

In part two of the study, A 2(Age) x 2 (Smoking) ANOVA on SOA25 and SOA5 showed a main effect of Age (SOA25: older adults $(\mathrm{M}=$ $94.55, \mathrm{SD}=154.95)$, younger adults $(\mathrm{M}=36.93, \mathrm{SD}=51.87), \mathrm{F}(1,100)$ $=7.8, \mathrm{p}<0.01$; SOA5: older adults $(\mathrm{M}=198.97, \mathrm{SD}=150.9)$, younger adults $(\mathrm{M}=90.79, \mathrm{SD}=51.9), \mathrm{F}(1,100)=30, \mathrm{p}<0.001)$. There were no other main effects or interactions (Figure 2, right).

Inhomogeneous Masks. Inhomogeneous masks were only tested in part one. An ANOVA on the factor Group showed no significant difference between groups for the gap grating $(\mathrm{F}(3,44)=1.44, \mathrm{p}=$ $0.244)$ or the $5-25$ grating $(\mathrm{F}(3,44)=0.65, \mathrm{p}=0.59)$. However, there was a significant main effect of group for the 25 grating $(\mathrm{F}(3.44)=$ $3.3, \mathrm{p}<0.05)$ (Figure 3, left). Post-hoc tests showed that non-smokers showed significantly less masking than deprived $+(\mathrm{t}(22)=1.8$, $\mathrm{p}<0.05)$ or non-deprived smokers $(\mathrm{t}(22)=2.5, \mathrm{p}<0.01)$; however, these effects did not survive a Bonferroni corrected alpha of 0.008 $(0.05 / 6)$. There was no difference between non-smokers and deprived smokers $(\mathrm{t}(22)=0.9, \mathrm{p}=0.18)$.

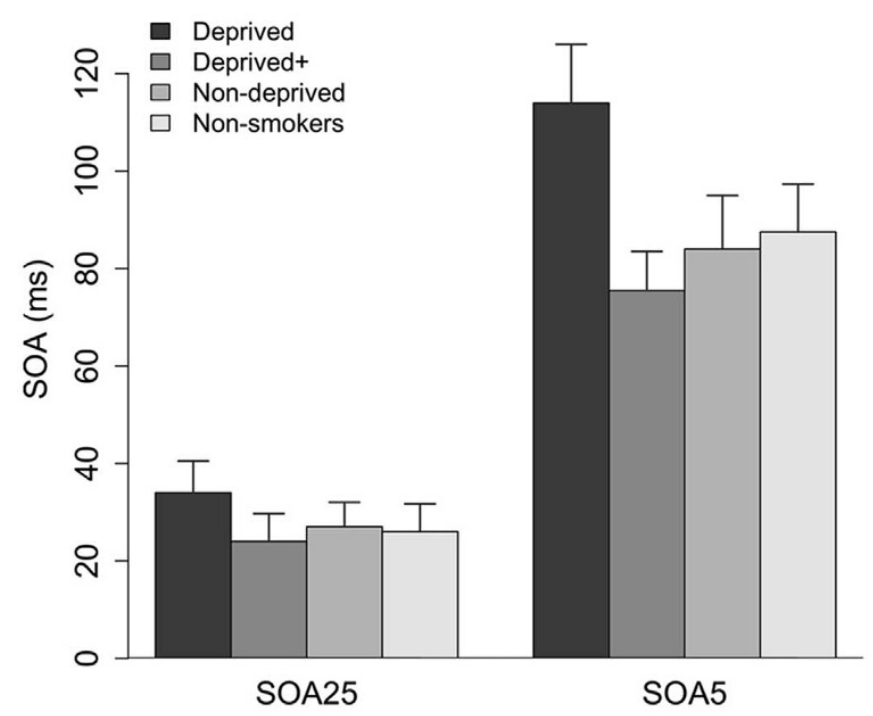

Fagerstrom Test of Nicotine Dependence. The Fagerstrom Test of Nicotine Dependence (FTND) ${ }^{47}$, was administered to all participants before the experiment. Participants in the smoking groups had range of FTND score from 5 to 10 and, on average, all smoking groups had scores above 6 (deprived: $M=6.9(0.5)$; Deprived $+: M=6.3(0.3)$; Non-deprived: $M=6.2(0.3)$ ), which is a score related to moderate to heavy smoking habits. A Pearson correlation showed no correlation between test scores and SOA (Figure 4).

\section{Discussion}

Abnormalities in the nicotinic system have been linked to schizophrenia. For example, the number of hippocampal nicotinic receptors is reduced in schizophrenic patients ${ }^{33}$ and changing the properties of nicotinic receptors has been suggested as a potential treatment for schizophrenia ${ }^{34,35}$. In vision research, Bakanidze et al. ${ }^{32}$ recently found that a mutation of the cholinergic nicotine receptor gene, alpha-7 subunit (CHRNA7) was correlated with schizophrenia and performance in the shine-through masking paradigm. Schizophrenic patients are often heavy smokers, which has been suggested to be a form of self-medication ${ }^{39-43}$. Given these relationships between nicotine, schizophrenia, and backward masking, we

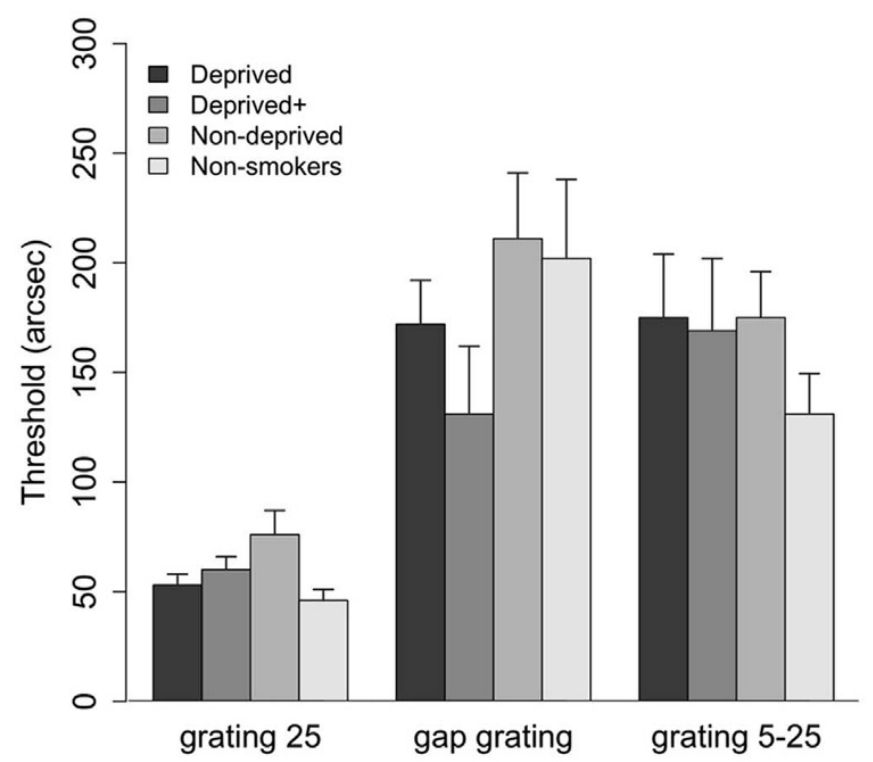

Figure 3 3 SOAs for 5- and 25- element gratings (left) for all participant groups. Error bars depict standard errors of the mean (SE). Deprived smokers showed significantly higher masking than deprived+ smokers in the SOA5 condition. Thresholds (arc sec) for the 25 grating, gap grating, and 5-25 grating (right). 

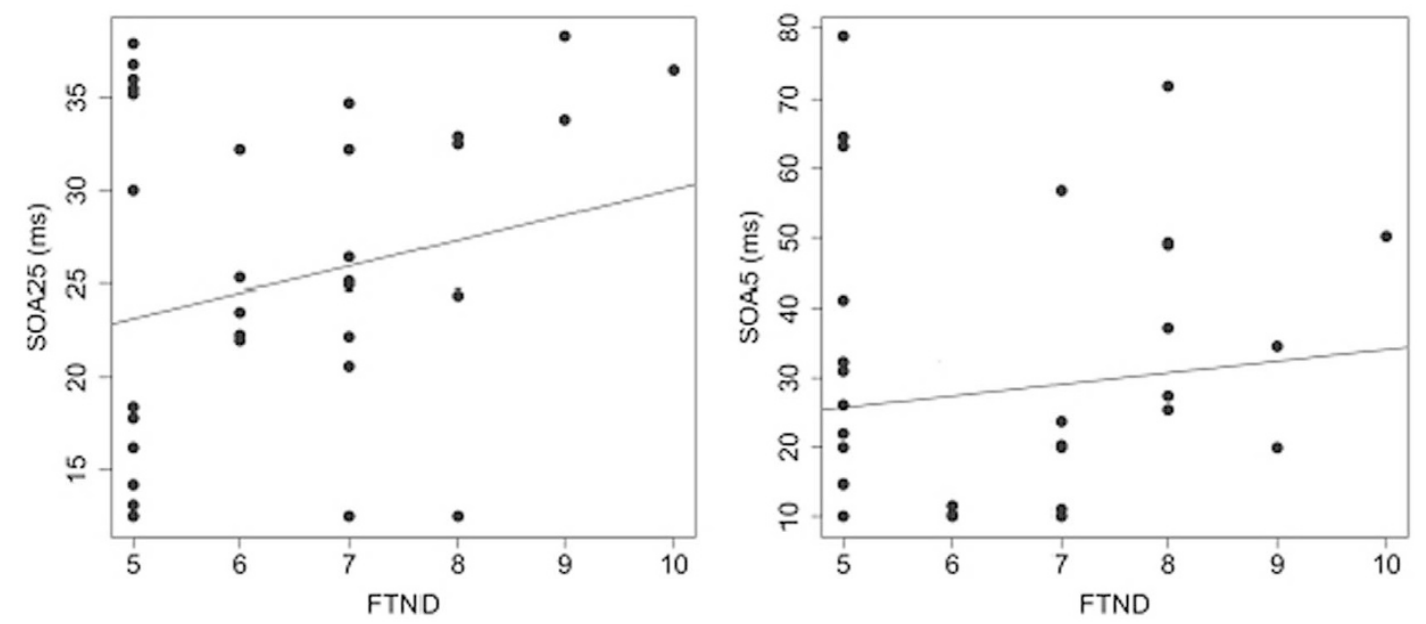

Figure $4 \mid$ Top: Correlations of the Fagerstrom Test of Nicotine Dependence score (FTND) with SOA25 (left) and SOA5 (right). None of the correlations is significant. Note the different scales of the $y$-axes.

investigated the relationship between nicotine and visual backward masking in healthy observers.

In general, we found only small effects of nicotine on masking. In the first part of this study, we tested non-smokers and three groups of smokers (deprived, non-deprived and deprived + ) in the shinethrough masking paradigm ${ }^{25-27}$. Only the comparison between stimulus onset asynchronies 5 (SOA5) in the deprived and the deprived + group was significant. In the second part of this study, we compared older and younger smokers and non-smokers. We found a main effect of age, in line with a previous study on backward masking and healthy ageing ${ }^{48}$, but no significant effect of smoking; if any, smokers performed better than controls.

For inhomogeneous masks, thresholds for the gap and the 5-25 grating were different from the 25 -element grating for all participant groups indicating intact spatiotemporal processing. The rationale is as follows: If spatial low pass filtering of the stimulus, for example, due to blurred vision, had decreased the visibility of the gap, we would have expected decreased thresholds, i.e., improved performance. Similarly, if temporal processing had been affected, we would have expected decreased thresholds for the 5-25 grating. Shinethrough does not occur for the 5-element grating ${ }^{25}$ and hence, good performance indicates that the shortly presented 5-element grating has not been processed efficiently. We found a significant main effect of smoking on the 25-element grating and post-hoc tests indicated that deprived + and non-deprived smokers showed more masking than non-smokers. However, the post-hoc tests did not survive Bonferroni correction. Future studies with larger sample sizes might need to clarify whether the main effect of group was due to noise or sample size. If the effects were true, it would indicate that smoking negatively affects temporal processing. If these effects were due to noise, it would indicate intact spatial and temporal visual processing across all groups, which again, is comparable to studies with schizophrenic patients who also have intact and fast perceptual grouping and figure-ground segmentation even though SOAS are a factor of 4 higher compared to controls ${ }^{26}$. The notion that nicotine consumption has no effect on visual processing is supported by a study of Colzato et al. $^{49}$, who showed that in healthy non-smokers nicotine consumption did not affect visual feature integration.

One reason for the small effects of smoking on masking might be that we deprived smokers for only 2 hours prior to the experiment. However, Hendricks et al. ${ }^{50}$ showed that nicotine-dependent smokers showed difficulties maintaining attention already 30 minutes after their latest cigarette. In addition, all smokers of our study were smoking at least 20 cigarettes per day and their scores on the Fagerstrom Test for Nicotine Dependence (FTND) ${ }^{47}$ were at or above 5, indicating a moderate to heavy level of nicotine dependence. Therefore, we expected a $2 \mathrm{~h}$ nicotine deprivation to be enough for inducing changes in nicotine-related mechanisms involved in backward masking.

Another reason for not finding performance differences between smokers and non-smokers might be that the nicotinic system of the smokers has adapted to the permanent supply of nicotine. This would also explain why we did not find superior performance in the deprived+ smokers compared to non-smokers. Interestingly, there seems to be a potential benefit of smoking in older adults, as seen in the second part of the study. Future studies with more participants are needed to clarify this. A more promising approach to test the effects of nicotine on backward masking might be to administer nicotine directly to non-smokers by using nicotine patches.

It has been suggested that nicotine is involved in visuospatial attention and inhibition. However, results vary tremendously between studies and it is difficult to generalize across studies given the difference in experimental procedures. Hahn et al. ${ }^{51}$, for example, administered nicotine or placebo patches to minimally deprived smokers and found that the administration of nicotine improved visuospatial attention. Vossel et al..$^{52}$ administered nicotine or placebo chewing gums to non-smoking participants and also found an advantage for participants that had received nicotine. Logeman et al. ${ }^{53}$, however, tested non-smokers and deprived smokers that had been administered with nicotine or placebo chewing gums before the start of the session and found no behavioural differences between groups in a visuospatial cueing paradigm. Similarly, Impey et al. ${ }^{54}$ administered nicotine or placebo chewing gums to non-smokers and also found no behavioural effects on spatial cueing. Taken together, these studies highlight the inconsistency in results following nicotine deprivation or administration to smokers and non-smokers on visuospatial cueing tasks and support our hypothesis that potential effects of smoking on visuospatial processing are small.

In conclusion, we only found a small relationship between nicotine deprivation and performance in backward masking. In addition, our results from part two suggest that nicotine has no negative long-term effect on backward masking performance.

\section{Methods}

Participants. Informed consent was obtained from all participants. All procedures involved in this study were approved by the Georgian National Bioethics Committee and were performed in accordance with the Declaration of Helsinki. All participants reported no mental, neural, or general health problems. All observers had normal or corrected-to-normal vision as determined by a score larger than 0.8 in the Freiburg visual acuity test ${ }^{55}$.

In part one of the study, 36 ( 18 female/18 male) cigarette smokers and 12 ( 6 female/ 6 male) non-smokers age from 20 to 40 years were recruited (mean age $=28.5$ years, 
$\mathrm{STD}=5$ years). Participants were asked to abstain from alcohol for 24 hrs and any other psychotropic drugs for $48 \mathrm{hrs}$ prior to the testing session.

All subjects took part in the Fagerstrom Test of Nicotine Dependence (FTND) ${ }^{47}$, which tests the level of nicotine addiction. To take part in the experiment observers had to score at least 5 in the FTND, indicating frequent smoking of about 20 cigarettes a day.

We tested 4 groups of participants: a) Nicotine deprived subjects who were not allowed to smoke 2 hours prior to testing and until the end of the experiment (deprived, $\mathrm{n}=12$, mean age $=27.3$ years, $\mathrm{STD}=5.3,6$ male); $\mathrm{b}$ ) Nicotine deprived subjects who were not allowed to smoke 2 hours prior to testing but had to smoke one cigarette right before the start of the experiment (deprived,$+ \mathrm{n}=12$, mean age $=25.7$ years, $\mathrm{STD}=4.35,6$ male); c) Non-deprived smokers who were allowed to smoke right until the start of the experiment (non-deprived, $\mathrm{n}=12$, mean age $=30.7$ years, STD $=4.95,6$ male); d) Non-smokers who had never smoked a cigarette (non-smokers, $\mathrm{n}=12$, mean age $=29.4$ years, STD $=5.67,6$ male). There was no difference in FTND scores between the three groups of smokers who took part in this experiment $(\mathrm{F}(2,33)=0.77, \mathrm{p}=0.47)$. There were also no significant age differences between all groups of participants $(\mathrm{F}(3,44)=2.49, \mathrm{p}=0.07)$.

In part two of the study, we compared 13 older smokers (mean age $=64$ years, STD $=2.4,5$ male, mean years of smoking $=43.1$, STD $=6.5), 24$ older non-smokers (mean age $=65.87$ years, $\mathrm{STD}=5.2,4$ male), 21 younger smokers $($ mean age $=22$ years, $\mathrm{STD}=2.4,11$ male, mean years of smoking $=5.4, \mathrm{STD}=2.1$ ) and 46 younger non-smokers (mean age $=21.8$ years, $\mathrm{STD}=2.5,9$ male). Older participants did not show any signs of mild cognitive impairment as tested with the Montreal Cognitive Assessment $(\mathrm{MoCa})^{56}$.

None of the participants from study two had been involved in part one of the study.

Stimuli and Apparatus. For both parts of the study, stimuli and apparatus were similar to Herzog et al. ${ }^{30}$.

The stimuli consisted of white vertical verniers and gratings comprising either 25 or 5 aligned verniers presented on a black background. A vertical vernier was composed of two bars that were slightly displaced in the horizontal direction either to the left or to the right. The length of a segment of the vernier, i.e. one bar, was $10 \mathrm{arc} \min$. Segments were separated by a small gap of 1 arc min. The aligned verniers of the gratings had the same lengths as the verniers. The horizontal distance between grating elements was about 3.33 arc min. The vernier and the central element of the grating always appeared in the middle of the screen. The stimuli appeared on a Samsung SyncMaster 957 DF CRT screen with a $100 \mathrm{~Hz}$ refresh rate. Maximal screen luminance was about $100 \mathrm{~cd} / \mathrm{m}^{2}$ as measured with a GretagMacbeth Eye-One Display 2 colorimeter. The background luminance on the screen was below $1 \mathrm{~cd} / \mathrm{m}^{2}$. Observers were seated in a dimly lit room at $5 \mathrm{~m}$ from the monitor.

Procedure. For both parts of the study, the procedure was similar to Herzog et al..$^{30}$. In the first step, we determined vernier duration (VD) using unmasked verniers. Verniers were presented in the middle of the screen and observers had to determine the offset direction (left or right) of the lower bar. For each observer, we determined the shortest VD for which the offset discrimination threshold $(75 \%$ correct discrimination) was below $40^{\prime \prime}$ (arc sec) ${ }^{29-32}$. Vernier duration was constant in each block and we varied the offset of the vernier using the adaptive PEST procedure. In the first block, verniers were presented for $150 \mathrm{~ms}$. In the following blocks, vernier duration was reduced when the threshold for offset discrimination was below the predefined value of $40^{\prime \prime}$ and increased when the threshold for offset discrimination was above $40^{\prime \prime}$. Each block consisted of 80 trials.

After the individual VD was determined, the nicotine-deprived + participants had to smoke one cigarette within 5 minutes. Five minutes after the intake of nicotine, we started with the next step of the experiment. Participants from the other three groups, non-smokers, non-deprived smokers and nicotine-deprived had a 10 minute break between step one and two. In the second step, individual vernier durations, as determined in step one, were used for each participant. We determined stimulusonset asynchronies (SOAs) for backward masking using the adaptive PEST proced$u_{r} e^{57}$. The SOA is defined as the difference between grating and vernier onset and is the sum of vernier duration and ISI (SOA = vernier duration + ISI). The grating was comprised of either 5 or 25 aligned verniers, i.e., verniers with out offset. The aligned verniers had the same lengths as the verniers. The horizontal distance between grating elements was about 3.33 arc min. The vernier and the central element of the grating always appeared in the middle of the screen and gratings lasted for $300 \mathrm{~ms}$. We adaptively assessed the stimulus-onset-asynchrony (SOA) between target and mask. For each observer, we used the individual vernier duration as determined in step one. The vernier offset size was set to $71^{\prime 29-32}$. The SOA was varied from trial to trial. We determined the critical SOA for which a performance level of $75 \%$ correct responses was obtained with a probit model using a standard maximum likelihood procedure $^{58,59}$. After each response, the SOA was adjusted based on the estimated model parameters until the model reached its terminus at $75 \%$ correct. The starting value of the SOA was set to $200 \mathrm{~ms}$. For each grating two thresholds were determined. The mean of the two thresholds was taken as the critical SOA. If observers were unable to reach a threshold value of $400 \mathrm{~ms}$ or below, a value of $450 \mathrm{~ms}$ was recorded (for details $\operatorname{see}^{26}$ ).

Only in part one, we included a third step, in which we tested performance for three inhomogeneous masks. We used the individual SOA and vernier durations determined in steps one and two. Therefore, baseline performance should be comparable across all observers. We determined individual vernier offset discrimination thresholds using the adaptive PEST strategy ${ }^{57}$. In the first condition, we presented the standard 25-element grating for $300 \mathrm{~ms}$ as described above. In the second condition, we presented a 25-element grating for $300 \mathrm{~ms}$, in which the middle two lines were removed (gap grating). The gap width was $250^{\prime \prime}$ and separated the central 5-elements from the peripheral $2 * 10$ elements. In the third condition, we presented a 25 -element grating for $280 \mathrm{~ms}$, which was preceded by a 5-element grating for $20 \mathrm{~ms}$ (5-25 grating). Hence, duration of the combined gratings was $300 \mathrm{~ms}$ as was the presentation time for conditions 1 and 2 .

1. Camm, A. J. et al. Heart rate variability: standards of measurement, physiological interpretation and clinical use. Task Force of the European Society of Cardiology and the North American Society of Pacing and Electrophysiology. Circulation 93, 1043-1065 (1999).

2. Solberg, Y., Rosner, M. \& Belkin, M. The association between cigarette smoking and ocular diseases. Surv. Ophthalmol. 42, 535-547 (1998).

3. Cheng, A. C. et al. The association between cigarette smoking and ocular diseases. Hong Kong Med. J. 6, 195-202 (2000).

4. Dalack, G. W., Healy, D. J. \& Meador-Woodruff, J. H. Nicotine dependence in schizophrenia: clinical phenomena and laboratory findings. Am. J. Psychiatry. 155, 1490-1501 (1998).

5. Dinas, P. C., Koutedakis, Y. \& Flouris, A. D. Effects of active and passive tobacco cigarette smoking on heart rate variability. Int. J. Cardiol. 163, 109-115 (2011).

6. Flouris, A. D., Vardavas, C. I., Metsios, G. S., Tsatsakis, A. M. \& Koutedakis, Y. Biological evidence for the acute health effects of secondhand smoke exposure. Am. J. Physiol. Lung. Cell Mol. Physiol. 298, L3-L12 (2010).

7. Galor, A. \& Lee, D. J. Effects of smoking on ocular health. Curr. Opin. Ophthalmol. 22, 477-482 (2011).

8. Giessing, C., Thiel, C. M., Rösler, F. \& Fink, G. R. The modulatory effects of nicotine on parietal cortex activity in a cued target detection task depend on cue reliability. Neuroscience. 137, 853-64. (2006).

9. Kumari, V. et al. Cognitive effects of nicotine in humans: an fMRI study. Neuroimage. 19, 1002-13 (2003).

10. Lawrence, N. S., Ross, T. J. \& Stein, E. A. Cognitive mechanisms of nicotine on visual attention. Neuron. 36, 539-48 (2002).

11. Mumenthaler, M. S. et al. Psychoactive drugs and pilot performance: a comparison of nicotine, donepezil, and alcohol effects. Neuropsychopharmacology. 28, 1366-73 (2003).

12. Rusted, J. M., Caulfield, D., King, L. \& Goode, A. Moving out of the laboratory: does nicotine improve everyday attention? Behav. Pharmacol. 11, 621-9 (2000)

13. Bell, S. L., Taylor, R. C., Singleton, E. G., Henningfield, J. E. \& Heishman, S. J. Smoking after nicotine deprivation enhances cognitive performance and decreases tobacco craving in drug abusers. Nicotine Tob. Res. 1, 45-52 (1999).

14. Warburton, D. M. \& Mancuso, G. Evaluation of the information processing and mood effects of a transdermal nicotine patch. Psychopharmacology. 135, 305-10 (1998).

15. Min, S. K., Moon, I. W., Ko, R. W. \& Shin, H. S. Effects of transdermal nicotine on attention and memory in healthy elderly non-smokers. Psychopharmacology. 159, 83-8 (2001).

16. Piper, M. E. et al. A multiple motives approach to tobacco dependence: The Wisconsin Inventory of Smoking Dependence Motives (WISDM-68). J. Consult. Clin. Psychol. 72, 139-154 (2004).

17. Russell, M. H., Peto, J. \& Patel, U. A. Classification of Smoking by Factorial Structure of Motives. J. R. Stat. Soc. Ser. A. Stat. Soc. 137, 313-346 (1974).

18. Wesnes, K. \& Warburton, D. M. Effects of smoking on rapid information processing performance. Neuropsychobiology. 9, 223-9 (1983).

19. Harrison, E. L., Coppola, S. \& McKee, S. A. Nicotine deprivation and trait impulsivity affect smokers' performance on cognitive tasks of inhibition and attention. Exp. Clin. Psychopharmacol. 17, 91-8 (2009).

20. Hatsukami, D. K., Hughes, J. R., Pickens, R. W. \& Svikis, D. Tobacco withdrawal symptoms: an experimental analysis. Psychopharmacology. 84, 231-6 (1984).

21. Hughes, J. R. \& Hatsukami, D. Signs and symptoms of tobacco withdrawal. Arch. Gen. Psychiatry. 43, 289-94 (1986).

22. Jacobsen, L. K. et al. Effects of smoking and smoking abstinence on cognition in adolescent tobacco smokers. Biol. Psychiatry. 57, 56-66 (2005).

23. McClernon, F. J. et al. Effects of smoking abstinence on adult smokers with and without attention deficit hyperactivity disorder: results of a preliminary study. Psychopharmacology. 197, 95-105 (2008).

24. Xu, J. et al. Brain activity in cigarette smokers performing a working memory task: effect of smoking abstinence. Biol. Psychiatry. 58, 143-50 (2005).

25. Herzog, M. H. \& Koch, C. Seeing properties of an invisible object: feature inheritance and shine-through. Proc. Natl. Acad. Sci. U S A. 98, 4271-5 (2001).

26. Herzog, M. H., Fahle, M. \& Koch, C. Spatial aspects of object formation revealed by a new illusion, shine-through. Vision Res. 41, 2325-35 (2001).

27. Herzog, M. H., Koch, C. \& Fahle, M. Shine-through: temporal aspects. Vision Res. 41, 2337-46 (2001).

28. Overney, L. S., Blanke, O. \& Herzog, M. H. (Enhanced temporal but not attentional processing in expert tennis players. PLoS One. 3, e2380 (2008).

29. Kunchulia, M., Pilz, K. S. \& Herzog, M. H. How alcohol intake affects visual temporal processing. Vision Res. 66, 1-6 (2012).

30. Herzog, M. H., Kopmann, S. \& Brand, A. Intact figure-ground segmentation in schizophrenia. Psychiatry Res. 129, 55-63 (2004). 
31. Chkonia, E. et al. The shine-through masking paradigm is a potential endophenotype of schizophrenia. PLoS One. 5, e14268 (2010).

32. Bakanidze, G. et al. Association of the nicotinic receptor a7 subunit gene (chrna7) with schizophrenia and visual backward masking. Front Psychiatry. 4, 133 (2013).

33. Freedman, R., Hall, M., Adler, L. E. \& Leonard, S. Evidence in postmortem brain tissue for decreased numbers of hippocampal nicotinic receptors in schizophrenia. Biol. Psychiatry. 38, 22-33 (1995).

34. Severance, E. G. \& Yolken, R. H. Novel alpha7 nicotinic receptor isoforms and deficient cholinergic transcription in schizophrenia. Genes Brain Behav. 7, 37-45 (2008).

35. Taly, A., Corringer, P. J., Guedin, D., Lestage, P. \& Changeux, J. P. Nicotinic receptors: allosteric transitions and therapeutic targets in the nervous system. Nat. Rev. Drug Discov. 8, 733-750 (2009).

36. de Leon, J. et al. Schizophrenia and smoking: an epidemiological survey in a state hospital. Am. J. Psychiatry. 152, 453-455 (1995).

37. DeBlack, S. Cigarette smoking as a risk factor for cataract and age-related macular degeneration: a review of the literature. Optometry. 74, 99-110 (2003).

38. Lasser, K. et al. Smoking and mental illness: A population-based prevalence study. JAMA. 284, 2606-2610 (2000).

39. Hahn, C. et al. Effects of smoking history on selective attention in schizophrenia. Neuropharmacology. 62, 1897-902 (2012).

40. Smith, R. C., Infante, M., Ali, A., Nigam, S. \& Kotsaftis, A. Effects of cigarette smoking on psychopathology scores in patients with schizophrenia: An experimental study. Subst. Abus. 22, 175-186 (2001).

41. Avila, M. T., Sherr, J. D., Hong, E., Myers, C. S. \& Thaker, G. K. Effects of nicotine on leading saccades during smooth pursuit eye movements in smokers and nonsmokers with schizophrenia. Neuropsychopharmacology. 28, 2184-2191 (2003).

42. Sacco, K. A. et al. Effects of cigarette smoking on spatial working memory and attentional deficits in schizophrenia: involvement of nicotinic receptor mechanisms. Arch. Gen. Psychiatry. 62, 649-659 (2005).

43. Leonard, S., Mexal, S. \& Freedman, R. Smoking, genetics and schizophrenia: Evidence for self-medication. J. Dual. Diagn. 3, 43-59 (2007).

44. Anstey, K. J., von Sanden, C., Salim, A. \& O’Kearney, R. Smoking as a risk factor for dementia and cognitive decline: a meta-analysis of prospective studies. Am. J. Epidemiol. 166, 367-78 (2007).

45. Hurley, S. F., Matthews, J. P. \& Guymer, R. H. Cost-effectiveness of smoking cessation to prevent age-related macular degeneration. Cost Eff. Resour. Alloc. 11, 6-18 (2008).

46. Murray, K. N. \& Abeles, N. Nicotine's effect on neural and cognitive functioning in an aging population. Aging Ment. Health. 6, 129-38 (2002).

47. Heatherton, T. F., Kozlowski, L. T., Frecker, R. C. \& Fagerstrom, K. O. The Fagerstrom test for nicotine dependence: a revision of the Fagerstrom tolerance questionnaire. Br. J. Addict. 86, 1119-1127 (1991).

48. Roinishvili, M., Chkonia, E., Stroux, A., Brand, A. \& Herzog, M. H. Combining vernier acuity and visual backward masking as a sensitive test for visual temporal deficits in aging research. Vision Res. 51, 417-23 (2011).

49. Colzato, S. L., Fagioli, S. \& Hommel, B. Caffeine, but not nicotine, enhances visual feature binding. Eur. J. Neurosc. 21, 591-595 (2005).

50. Hendricks, P., Ditre, J., Drobes, D. \& Brandon T. The early time course of smoking withdrawal effects. Psychopharmacology. 187, 385-396 (2006).
51. Hahn, B. et al. Nicotine enhances visuospatial attention by deactivating areas of the resting brain default network. J. Neurosci. 27, 3477-89 (2007).

52. Vossel, S., Thiel, C. M. \& Fink, G. R. (2008).Behavioral and neural effects of nicotine on visuospatial attentional reorienting in non-smoking subjects. Neuropsychopharmacology. 33, 731-8 (2008).

53. Logemann, H. N., Böcker, K. B., Deschamps, P. K., Kemner, C. \& Kenemans, J. L. Differences between nicotine-abstinent smokers and non-smokers in terms of visuospatial attention and inhibition before and after single-blind nicotine administration. Neuroscience. 26, 375-82 (2014).

54. Impey, D., Chique-Alfonzo, M., Shah, D., Fisher, D. J. \& Knott, V. J. Effects of nicotine on visuospatial attentional orienting in non-smokers. Pharmacol Biochem Behav. 106, 1-7 (2013)

55 . Bach, M. The Freiburg visual acuity test-automatic measurement of visual acuity. Optom. Vis. Sci. 73, 49-53 (1996).

56. Nasreddine, Z. S. et al. The Montreal Cognitive Assessment, MoCA: a brief screening tool for mild cognitive impairment. J. Am. Geriatr. Soc. 53, 695-9 (2005).

57. Creelman, C. D. \& Taylor, M. M. Some pitfalls in adaptive testing: comments on "temporal integration and periodicity pitch" J. Acoust. Soc. Am. 46, 1581-2 (1996).

58. Finney, D. J. Probit Analysis. (Cambridge University Press, Cambridge, 1971).

59. Finney, D. J. Statistical Method in Biological Assay. (Charles Griffin and Co., London and High Wycombe, 1978).

\section{Acknowledgments}

We would like to thank Marc Repnow for his help setting up the experiments. This project was funded by the research grant "Perception, cognition and healthy ageing" of the VELUX foundation.

\section{Author contributions}

M.K. collected the data. All authors have designed the study and wrote the manuscript. K.P. contributed in-depth aging expertise. M.H. contributed in-depth backward masking expertise. Michael Herzog is a member of the NCCR SYNAPSY of the Swiss National Science Foundation (SNF)

\section{Additional information}

Competing financial interests: The authors declare no competing financial interests.

How to cite this article: Kunchulia, M., Pilz, K.S. \& Herzog, M.H. Small effects of smoking on visual spatiotemporal processing. Sci. Rep. 4, 7316; DOI:10.1038/srep07316 (2014).

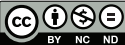

This work is licensed under a Creative Commons Attribution-NonCommercialNoDerivs 4.0 International License. The images or other third party material in this article are included in the article's Creative Commons license, unless indicated otherwise in the credit line; if the material is not included under the Creative Commons license, users will need to obtain permission from the license holder in order to reproduce the material. To view a copy of this license, visit http:// creativecommons.org/licenses/by-nc-nd/4.0/ 